\title{
Commentary on "femoral nerve block prevents deep venous thrombosis of the lower extremity after knee arthroplasty: a single-center randomized controlled trial"
}

\author{
Brian Wu, Ido Badash* \\ Keck School of Medicine of University of Southern California (USC), Los Angeles, CA, USA \\ *Correspondence to: Brian Wu, M.D., brianwwu@usc.edu. \\ orcid: 0000-0002-5418-5245 (Brian Wu)
}

\section{Abstract}

Total knee arthroplasty (TKA) is the most frequently performed surgical remediation for patients with long-standing osteoarthritis, but a variety of negative outcomes following TKA have been noted, including deep vein thrombosis (DVT). It is the aim of Chen et al.'s randomized controlled trial to explore the efficacy of advanced ultra-sounded guided femoral nerve blocks for reducing serum inflammatory cytokine levels associated with DVT. By specifically measuring changes in neurtrophil elastase following use of either an advanced femoral nerve block 30 minutes prior to surgery or conventional femoral nerve block after surgery and intravenous analgesia, Chen et al.'s study will elucidate the most reliable method for preventing DVT occurrence after TKA. While this commentary identifies several limitations to the study that must be addressed, the sound study design, adequate number of participants and the potential impact of the work on improving patient outcomes from a common procedure make it a valuable contribution to the field of orthopedics. With conclusive results from this study, as well as additional studies investigating the benefits of advanced femoral nerve blocks on outcomes like muscle atrophy, the procedure could become an important consideration during TKA surgery.

Key words: knee arthroplasty; femoral nerve block; deep venous; serum inflammatory cytokine

doi: 10.4103/2468-5674.183007

How to cite this article: Wu B, Badash I (2016) Commentary on "femoral nerve block prevents deep venous thrombosis of the lower extremity after knee arthroplasty: a single-center randomized controlled trial". Clin Transl Orthop 1(2):83-84.

This randomized controlled trial reported by Chen et al. (2016), exploring the influence of advanced femoral nerve blocks on reducing serum inflammatory cytokine levels associated with deep vein thrombosis (DVT) after total knee arthroplasty (TKA), is both timely and relevant for orthopedic surgeons who desire to improve patient outcomes after performing this common procedure. TKA is the most frequently performed surgical remediation for patients with long-standing osteoarthritis, but a variety of negative outcomes following the surgery have been noted, including persistent muscle atrophy, polyethylene wear, infection and DVT (Healy et al., 2013; Kim et al., 2014). DVT is a particularly significant outcome, with a reported incidence as high as $36.9 \%$ in bilateral TKA even with the use of prophylaxis (O'Reily et al., 2005), and Chen et al. (2016) hypothesize that advanced femoral nerve blocks will limit this event.
The authors' chosen study design has several strengths. First, plasma inflammatory cytokine levels will be measured at several regular postoperative intervals, demonstrating the changes in DVT-promoting cytokines over time. Additionally, collecting samples up to $12-48$ hours post-surgery will provide a greater window of time to detect these changes. The authors' selection of study arms, as comparing outcomes between patients receiving a nerve block both preand post-operatively, and patients receiving intravenous analgesia, will be effective and useful in clarifying the best use of femoral nerve blocks for preventing the occurrence of DVT. Finally, this trial will enroll a relatively large sample size of 120 patients, although a power analysis should be included to determine how many participants will be needed to obtain statistically significant results.

The study also has room for improvement. As stated in the background, ischemia-reperfusion injury associated 
with tourniquet use is an important event leading to the release of inflammatory markers and the development of DVT. While the authors will measure plasma cytokine levels as a proxy for determining relative differences in the inflammatory states of patients, they do not have a way of determining patients' ischemic states. Given that recent studies have introduced plasma metabolites as strong indicators of ischemia following TKA surgery (Ejaz et al., 2015), the authors should consider performing an analysis of these metabolites (glucose, lactate, pyruvate, glycerol, etc.) before and after TKA, along with inflammatory cytokines, in order to strengthen the validity of their argument that ischemia-reperfusion injury will lead to neutrophil elastase release and subsequent DVT. Additionally, it is unclear from the study protocol where exactly the plasma cytokine collections will occur. The authors should ensure that the cytokine levels are measured within the ischemic zone of the tourniquet in order to obtain the most direct inflammatory changes following the surgery.

While the study is a good starting point for highlighting the benefits of advanced femoral nerve blocks on preventing DVT, recent studies have questioned whether tourniquet use during TKA and subsequent inflammation are associated with an increased risk of DVT at all. In fact, a study by Abbas et al. (2013) has suggested that tourniquet use during TKA is associated with increased fibrinolytic activity immediately after the surgical procedure, and another study has found that tourniquet use does not increase the risk of DVT (Fukuda et al., 2007).

Therefore, future studies might consider focusing on a more well understood complication of TKA in investigating the benefits of advanced femoral nerve blocks on inflammatory changes. For example, persistent muscle atrophy following tourniquet use in TKA is perhaps an even more common and preventable outcome of these surgeries (Mizner et al., 2005a). Several studies have shown that tourniquet use is associated with changes in inflammatory cytokines indicative of ischemia, reperfusion injury and muscle atrophy (Mizner et al., 2005b; Vignaud et al., 2010; Tran et al., 2011). Chen et al. (2016) have already included muscle strength rating as a secondary outcome of their study, and so future studies by the authors might choose to investigate the role of advanced femoral nerve blocks in preventing inflammatory and subsequent muscular changes, providing further value to the use of this analgesic procedure during TKA surgery.

Overall, this randomized controlled trial by Chen et al.
(2016) is a valuable contribution to the field of orthopedics, investigating a potential way to limit a significant negative outcome of a common procedure. The study design of the trial is sound, the number of participants is adequate, and the potential impact of the work is high enough to alter the view on the role of advanced femoral nerve blocks in TKA surgeries. With conclusive results from this study, as well as additional studies investigating the benefits of advanced femoral nerve blocks on outcomes like muscle atrophy, the procedure could become an important consideration during TKA surgery.

\section{References}

Abbas K, Raza H, Umer M, Hafeez K (2013) Effect of early release of tourniquet in total knee arthroplasty. J Coll Physicians Surg Pak 23:562-565.

Chen Y, Qu S, Ma G, Meng JH, Ni XL (2016) Femoral nerve block prevents deep venous thrombosis of the lower extremity after knee arthroplasty: a single-center randomized controlled trial. Clin Transl Orthop 1:8-14.

Ejaz A, Laursen AC, Kappel A, Jakobsen T, Nielsen PT, Rasmussen $\mathrm{S}$ (2015) Tourniquet induced ischemia and changes in metabolism during TKA: a randomized study using microdialysis. BMC Musculoskelet Disord 16:326.

Fukuda A, Hasegawa M, Kato K, Shi D, Sudo A, Uchida A (2007) Effect of tourniquet application on deep vein thrombosis after total knee arthroplasty. Arch Orthop Trauma Surg127:671-675.

Healy WL, Della Valle CJ, Iorio R, Berend KR, Cushner FD, Dalury DF, Lonner JH (2013) Complications of total knee arthroplasty: standardized list and definitions of the Knee Society. Clin Orthop Relat Res 471:215-220.

Kim KT, Lee S, Ko DO, Seo BS, Jung WS, Chang BK (2014) Causes of failure after total knee arthroplasty in osteoarthritis patients 55 years of age or younger. Knee Surg Relat Res 26:13-19.

Mizner RL, Petterson SC, Snyder-Mackler L (2005a) Quadriceps strength and the time course of functional recovery after total knee arthroplasty. J Orthop Sports Phys Ther 35:424-436.

Mizner RL, Petterson SC, Stevens JE, Vandenborne K, SnyderMackler L (2005b) Early quadriceps strength loss after total knee arthroplasty. The contributions of muscle atrophy and failure of voluntary muscle activation. J Bone Joint Surg Am 87:1047-1053.

O'Reily RF, Burgess IA, Zicat B (2005) The prevalence of venous thromboembolism after hip and knee replacement surgery. Med J Aust 182:154-159.

Tran TP, Tu H, Pipinos II, Muelleman RL, Albadawi H, Li Y-L (2011) Tourniquet-induced acute ischemia-reperfusion injury in mouse skeletal muscles: involvement of superoxide. Eur J Pharmacol 650:328-334.

Vignaud A, Hourde C, Medja F, Agbulut O, Butler-Browne G, Ferry A (2010) Impaired skeletal muscle repair after ischemia-reperfusion injury in mice. J Biomed Biotechnol 2010:724914. 